\title{
Estimation of Stress-Strength Reliability for the Generalized Pareto Distribution Based on Progressively Censored Samples
}

\author{
S. Rezaei ${ }^{1}$ - R. Alizadeh Noughabi ${ }^{1}$. \\ S. Nadarajah ${ }^{2}$
}

Received: 18 February 2015 / Revised: 20 March 2015 / Accepted: 23 March 2015 /

Published online: 3 April 2015

(C) Springer-Verlag Berlin Heidelberg 2015

\begin{abstract}
This paper deals with the estimation of stress-strength reliability parameter, $R=P(Y<X)$, based on progressively type II censored samples when stress, strength are two independent generalized Pareto random variables. The maximum likelihood estimators, their asymptotic distributions, asymptotic confidence intervals, bootstrap based confidence intervals and Bayes estimators are derived for $R$. Using Monte Carlo simulations, the MSE, Bayes risk estimators, credible sets and coverage probabilities are computed and compared.
\end{abstract}

Keywords Generalized Pareto distribution - Maximum likelihood estimator . Progressively type II censored samples · Uniformly minimum variance unbiased estimator

\section{Introduction}

Estimation of $R=P(Y<X)$ is widely known as stress-strength modeling: if $X$ denotes the stress that a system is subjected to and $Y$ the strength of the system then $R$ is the probability of the failure of the system. Many authors have discussed parametric and non-parametric inference on $R$ when complete random samples are available on each $X$ and $Y$. We mention: [16] for the case $X, Y$ are independent gamma random variables; [4] for the case $X, Y$ are independent exponential random variables with common scale; [17] for the case $X, Y$ are independent logistic random variables; [18] for the case $X, Y$ are independent Laplace random variables; [12]

\footnotetext{
$凶 \quad$ S. Rezaei

srezaei@aut.ac.ir

1 Department of Statistics, Amirkabir University of Technology, Tehran, Iran

2 School of Mathematics, University of Manchester, Manchester, United Kingdom
} 
for the case $X, Y$ are independent generalized exponential random variables; [19] for the case $X, Y$ are independent beta random variables; [20] for the case $X, Y$ are dependent gamma random variables; [15] for the case $X, Y$ are independent Burr type III random variables; [13] for the case $X, Y$ are independent Weibull random variables; [21] for the case $X, Y$ are dependent exponential random variables; [11] for the case $X, Y$ are independent exponential random variables; [24] for the case $X, Y$ are independent Gompertz random variables; [22] for the case $X, Y$ are independent generalized exponential random variables; [23] for the case $X, Y$ are independent generalized Pareto random variables; [1] for the case $X, Y$ are independent generalized logistic random variables. We refer the readers to [10] for a review of other papers.

There has been little work on parametric and non-parametric inference of $R=$ $P(Y<X)$ when samples available on $X$ and $Y$ are not complete. In reliability studies, the experimenter may not always obtain complete information on failure times for all experimental units. Some real life examples giving censored data are: (i) sometimes a failure is planned and expected but does not occur due to operator error, equipment malfunction, test anomaly, etc; (ii) sometimes engineers plan a test program so that, after a certain time limit or number of failures, all other tests will be terminated. Among various censoring schemes, the type II progressive censoring scheme has become very popular. It can be described as follows: Let $N$ items be put in a life time study and $n(<N)$ items be completely observed; At the time of the first failure, $r_{1}$ surviving units are removed from the $N-1$ remaining items; At the time of the next failure, $r_{2}$ items are randomly withdrawn from the $N-r_{1}-2$ remaining items; When the $n$th failure occurs all the remaining $N-n-r_{1}-\cdots-r_{n-1}$ items are removed. See [2] for more details.

The aim of this paper is to extend the results in [23] for progressively type II censored samples, i.e., we develop inference procedures for $R=P(Y<X)$ when $X$ and $Y$ are independent generalized Pareto random variables and samples available on them are progressively type II censored.

The contents of this paper are organized as follows: the generalized Pareto distribution is introduced in Sect. 2; estimation of $R$ when $X$ and $Y$ are generalized Pareto random variables with common scale parameter is addressed in Sect. 3, giving the maximum likelihood estimator (MLE), its asymptotic distribution and bootstrap confidence intervals for $R$; estimation of $R$ when the common scale parameter is known is addressed in Sect. 4, giving maximum likelihood, uniformly minimum variance unbiased and Bayes estimators of $R$; estimation of $R$ in the general case is addressed in Sect. 5, giving maximum likelihood and Bayes estimators of $R$; Monte Carlo simulations are used to compare these estimators in Sect. 6.

The original contributions of this paper are Sects. 3 to 6. These include the algorithm presented in Sect. 4.4.

\section{Preliminaries}

A random variable $X$ is said to have the generalized Pareto (GP) distribution if its probability density function (pdf) is given by 


$$
f_{X}(x)=\frac{1}{\sigma}\left(1+\xi \frac{x-\mu}{\sigma}\right)^{-\left(\frac{1}{\xi}+1\right)}
$$

for $\xi \in \Re, \mu \in \Re$ and $\sigma>0$. Exponential, Pareto and logistic distributions are special cases of the GP distribution. The GP distribution has the decreasing failure rate (DFR) property.

A reparametrization of the GP distribution has the pdf and cumulative distribution function (cdf) specified by

$$
f_{X}(x)=\alpha \lambda(1+\lambda x)^{-(\alpha+1)}
$$

and

$$
F_{X}(x)=1-(1+\lambda x)^{-\alpha},
$$

respectively, for $x>0, \alpha>0$ and $\lambda>0$. The parameter $\alpha$ is a shape parameter and $\lambda$ is a scale parameter.

We shall write $X \sim \operatorname{GP}(\alpha, \lambda)$ if $X$ has the pdf and cdf specified by (2) and (3), respectively. Let $X \sim \mathrm{GP}\left(\alpha, \lambda_{1}\right)$ and $Y \sim \mathrm{GP}\left(\beta, \lambda_{2}\right)$ be independent random variables. The aim of this paper is to estimate $R=P(Y<X)$ based on progressively type II censored samples. We consider three cases.

\section{Estimation of $\boldsymbol{R}$ with Same Scale Parameter}

Here, we investigate properties of $R$ when $\lambda_{1}=\lambda_{2}=\lambda$. In this case,

$$
R=P(Y<X)=\frac{\beta}{\alpha+\beta}
$$

\subsection{Maximum Likelihood Estimator}

Suppose $X_{1: N}, X_{2: N}, \ldots, X_{n: N}$ is a progressively type II censored sample from $G P(\alpha, \lambda)$ and $Y_{1: M}, Y_{2: M}, \ldots, Y_{m: M}$ a progressively type II censored sample from $G P(\beta, \lambda)$. The likelihood function is (see [3])

$$
L(\alpha, \beta, \lambda)=K_{1} K_{2} \prod_{i=1}^{n} f_{X}\left(x_{i}\right)\left[1-F_{X}\left(x_{i}\right)\right]^{r_{i}} \times \prod_{j=1}^{m} f_{Y}\left(y_{j}\right)\left[1-F_{Y}\left(y_{j}\right)\right]^{r^{\prime}{ }_{i}}
$$

where

$$
\begin{aligned}
& K_{1}=\left(N-1-r_{1}\right)\left(N-2-r_{1}-r_{2}\right) \cdots\left(N-n+1-r_{1}-\cdots-r_{n-1}\right), \\
& K_{2}=M\left(M-1-r_{1}^{\prime}\right)\left(M-2-r_{1}^{\prime}-r_{2}^{\prime}\right) \cdots\left(M-m+1-r_{1}^{\prime}-\cdots-r_{m-1}^{\prime}\right),
\end{aligned}
$$

and $r, r^{\prime}$ denote the number of items randomly withdrawn from samples corresponding to $X$ and $Y$, respectively. 
By (2) and (3), (5) can be expressed as

$$
\begin{aligned}
L(\alpha, \beta, \lambda)= & K_{1} K_{2} \prod_{i=1}^{n} \alpha \lambda\left(1+\lambda x_{i}\right)^{-(\alpha+1)}\left(1+\lambda x_{i}\right)^{-\alpha r_{i}} \\
& \times \prod_{j=1}^{m} \beta \lambda\left(1+\lambda y_{j}\right)^{-(\beta+1)}\left(1+\lambda y_{j}\right)^{-\beta r_{j}^{\prime}} \\
= & K_{1} K_{2} \lambda^{n+m} \alpha^{n} \beta^{m} \prod_{i=1}^{n}\left(1+\lambda x_{i}\right)^{-\alpha\left(1+r_{i}\right)+1} \\
& \times \prod_{j=1}^{m}\left(1+\lambda y_{j}\right)^{-\beta\left(1+r_{j}^{\prime}\right)-1} .
\end{aligned}
$$

The log likelihood function is

$$
\begin{aligned}
\ell(\alpha, \beta, \lambda)= & \log K_{1}+\log K_{2}+(n+m) \log \lambda+n \log \alpha+m \log \beta \\
& -\sum_{i=1}^{n}\left[\alpha\left(1+r_{i}\right)+1\right] \log \left(1+\lambda x_{i}\right) \\
& -\sum_{j=1}^{m}\left[\beta\left(1+r_{j}^{\prime}\right)+1\right] \log \left(1+\lambda y_{j}\right) .
\end{aligned}
$$

The MLEs of parameters can be derived as the simultaneous solutions of

$$
\begin{aligned}
& \frac{\partial \ell(\alpha, \beta, \lambda)}{\partial \alpha}=\frac{n}{\alpha}-\sum_{i=1}^{n}\left(1+r_{i}\right) \log \left(1+\lambda x_{i}\right), \\
& \frac{\partial \ell(\alpha, \beta, \lambda)}{\partial \beta}=\frac{m}{\beta}-\sum_{j=1}^{m}\left(1+r_{j}^{\prime}\right) \log \left(1+\lambda y_{j}\right)
\end{aligned}
$$

and

$$
\frac{\partial \ell(\alpha, \beta, \lambda)}{\partial \lambda}=\frac{m+n}{\lambda}-\sum_{i=1}^{n} \frac{\alpha\left[\left(1+r_{i}\right)+1\right] x_{i}}{1+\lambda x_{i}}-\sum_{j=1}^{m} \frac{\beta\left[\left(1+r_{j}^{\prime}\right)+1\right] y_{j}}{1+\lambda y_{j}}
$$

Rearranging (13)-(15), we can obtain the MLEs of $\alpha$ and $\beta$ as

$$
\begin{aligned}
\widehat{\alpha} & =\frac{n}{\sum_{i=1}^{n}\left(1+r_{i}\right) \log \left(1+\lambda x_{i}\right)}, \\
\widehat{\beta} & =\frac{m}{\sum_{j=1}^{m}\left(1+r_{j}^{\prime}\right) \log \left(1+\lambda y_{j}\right)} .
\end{aligned}
$$


The MLE of $\lambda$ can be obtained as the root of

$$
\begin{gathered}
(n+m)\left[\frac{n}{\sum_{i=1}^{n}\left(1+r_{i}\right) \log \left(1+\lambda x_{i}\right)} \sum_{i=1}^{n} \frac{\left(1+r_{i}\right) x_{i}}{1+\lambda x_{i}}-\sum_{i=1}^{n} \frac{x_{i}}{1+\lambda x_{i}}\right. \\
\left.-\frac{m}{\sum_{j=1}^{m}\left(1+r_{j}^{\prime}\right) \log \left(1+\lambda y_{j}\right)} \sum_{j=1}^{m} \frac{\left(1+r_{j}^{\prime}\right) y_{j}}{1+\lambda y_{j}}-\sum_{j=1}^{m} \frac{y_{j}}{1+\lambda y_{j}}\right]^{-1}=0 .
\end{gathered}
$$

\subsection{Asymptotic Distribution}

Here, the asymptotic distribution of $\widehat{\boldsymbol{\theta}}=(\widehat{\alpha}, \widehat{\beta}, \widehat{\lambda})$ and the asymptotic distribution of $\widehat{R}$ are derived. The latter can be used to derive an asymptotic confidence interval of $R$ and this in turn requires the Fisher information matrix of $\boldsymbol{\theta}=(\alpha, \beta, \lambda)$.

If $X_{1: N}, X_{2: N}, \ldots, X_{n: N}$ is a progressively type II censored sample from $G P(\alpha, \lambda)$ with censored scheme $\left(r_{1}, r_{2}, \ldots, r_{n}\right)$, then $Z_{1: N}, Z_{2: N}, \ldots, Z_{n: N}$ is a progressively type II censored sample from the unit exponential distribution with censored scheme $\left(r_{1}, r_{2}, \ldots, r_{n}\right)$, where $Z_{i: N}=\alpha \log \left(1+\lambda X_{i: N}\right)$. By [3],

$$
E\left[\log \left(1+\lambda X_{i: N}\right)\right]=\frac{1}{\alpha} E\left(Z_{i: N}\right)=\frac{1}{\alpha} \sum_{k=1}^{i} \frac{1}{N-\left(\sum_{s=0}^{k-1} r_{s}\right)-k+1}
$$

for $i=1,2, \ldots, n$. By [9], the pdf of $X_{i: N}$ is

$$
f_{X_{i: N}}(x)=c_{i-1} \sum_{k=1}^{i} a_{k, i} \alpha \lambda\left(1+\lambda x_{i}\right)^{-\left(\alpha \gamma_{k}+1\right)},
$$

where

$$
\begin{aligned}
& \gamma_{k}=N-k+1+\sum_{s=k}^{n} r_{s}, \\
& a_{k, i}=\prod_{\substack{s=1 \\
s \neq k}}^{i} \frac{1}{\gamma_{s}-\gamma_{i}} .
\end{aligned}
$$

The expected Fisher information matrix is

$$
I(\theta)=-\left[\begin{array}{lll}
E\left(\frac{\partial^{2} \ell}{\partial \alpha^{2}}\right) & E\left(\frac{\partial^{2} \ell}{\partial \alpha \partial \beta}\right) & E\left(\frac{\partial^{2} \ell}{\partial \alpha \partial \lambda}\right) \\
E\left(\frac{\partial^{2} \ell}{\partial \beta \partial \alpha}\right) & E\left(\frac{\partial^{2} \ell}{\partial \beta^{2}}\right) & E\left(\frac{\partial^{2} \ell}{\partial \beta \partial \lambda}\right) \\
E\left(\frac{\partial^{2} \ell}{\partial \lambda \partial \alpha}\right) & E\left(\frac{\partial^{2} \ell}{\partial \lambda \partial \beta}\right) & E\left(\frac{\partial^{2} \ell}{\partial \lambda^{2}}\right)
\end{array}\right]=\left[\begin{array}{ccc}
I_{11} & I_{12} & I_{13} \\
I_{21} & I_{22} & I_{23} \\
I_{31} & I_{32} & I_{33}
\end{array}\right] .
$$


By using

$$
\int_{0}^{\infty} x^{r-1}(1+\gamma x)^{-\delta} d x=\gamma^{-r} B(r, \delta-r)
$$

for $0<r<\delta$, where $B(x, y)$ denotes the beta function, we obtain

$$
\begin{gathered}
I_{11}=\frac{n}{\alpha^{2}}, \\
I_{12}=I_{21}=0, \\
I_{22}=\frac{m}{\beta^{2}}, \\
I_{13}=I_{31}=\sum_{i=1}^{n}\left(1+r_{i}\right) E\left(\frac{X_{i}}{1+\lambda X_{i}}\right) \\
=\frac{\alpha}{\lambda} \sum_{i=1}^{n}\left(1+r_{i}\right) \sum_{k=1}^{i} a_{k, i} c_{i-1} B\left(2, \alpha \gamma_{k}\right) .
\end{gathered}
$$

Since

$$
\begin{aligned}
E\left(\frac{X_{i}}{1+\lambda X_{i}}\right) & =\int_{0}^{\infty} \frac{x}{1+\lambda x} c_{i-1} \sum_{k=1}^{i} a_{k, i} \alpha \lambda(1+\lambda x)^{-\left(\alpha \gamma_{k}+1\right)} d x \\
& =\sum_{k=1}^{i} \alpha \lambda a_{k, i} c_{i-1} \int_{0}^{\infty} x(1+\lambda x)^{-\left(\alpha \gamma_{k}+2\right)} d x \\
& =\sum_{k=1}^{i} \alpha \lambda a_{k, i} c_{i-1} \lambda^{-2} B\left(2, \alpha \gamma_{k}\right)
\end{aligned}
$$

we obtain

$$
\begin{aligned}
& I_{23}=I_{32}=\frac{\beta}{\lambda} \sum_{j=1}^{m}\left(1+r_{j}^{\prime}\right) c_{i-1} \sum_{k=1}^{i} a_{k, i} B\left(2, \beta \gamma_{k}\right), \\
& I_{33}=\frac{1}{\lambda^{2}}\left\{(n+m)-\alpha \sum_{i=1}^{n}\left[\alpha\left(1+r_{i}\right)+1\right] c_{i-1} \sum_{k=1}^{i} a_{k, i} B\left(3, \alpha \gamma_{k}\right)\right. \\
& \left.-\beta \sum_{j=1}^{m}\left[\beta\left(1+r_{j}^{\prime}\right)+1\right] c_{i-1} \sum_{k=1}^{i} a_{k, i} B\left(3, \beta \gamma_{k}\right)\right\} \text {. }
\end{aligned}
$$

Theorem 1 now follows.

Theorem 1 As $n \rightarrow \infty$ and $m \rightarrow \infty$,

$$
[\sqrt{n}(\widehat{\alpha}-\alpha), \sqrt{m}(\widehat{\beta}-\beta), \sqrt{n}(\widehat{\lambda}-\lambda)] \rightarrow N_{3}\left(\mathbf{0}, \mathbf{A}^{-1}(\alpha, \beta, \lambda)\right),
$$


where

$$
\mathbf{A}(\alpha, \beta, \lambda)=\left[\begin{array}{lll}
a_{11} & 0 & a_{13} \\
0 & a_{22} & a_{23} \\
a_{31} & a_{32} & a_{33}
\end{array}\right]
$$

with

$$
\begin{gathered}
a_{11}=\frac{1}{n} I_{11}, \\
a_{22}=\frac{1}{m} I_{22}, \\
a_{13}=a_{31}=\frac{1}{n} I_{13}, \\
a_{23}=a_{32}=\frac{1}{\sqrt{n m}} I_{23}, \\
a_{33}=\frac{1}{n} I_{33} .
\end{gathered}
$$

Theorem 2 As $n \rightarrow \infty, m \rightarrow \infty$ and $\frac{n}{m} \rightarrow p$,

$$
\sqrt{n}(\widehat{R}-R) \rightarrow N(0, B)
$$

where

$$
B=\frac{1}{c(\alpha+\beta)^{4}}\left[\beta^{2}\left(a_{22} a_{33}-a_{23}^{2}\right)-2 \alpha \beta \sqrt{p} a_{23} a_{31}+\alpha^{2} p\left(a_{11} a_{33}-a_{13}^{2}\right)\right],
$$

and $c=a_{11} a_{22} a_{33}-a_{11} a_{23} a_{32}-a_{13} a_{22} a_{31}$.

\section{Estimation of $\boldsymbol{R}$ When $\lambda$ is Known}

Without loss of generality we can assume that $\lambda=1$. It is clear that

$$
R=P(Y<X)=\frac{\beta}{\alpha+\beta}
$$

\subsection{Maximum Likelihood Estimator of $\boldsymbol{R}$}

Let $X_{1: N}, X_{2: N}, \ldots, X_{n: N}$ be a progressively type II censored sample from $G P(\alpha, 1)$ and $Y_{1: M}, Y_{2: M}, \ldots, Y_{m: M}$ be a progressively type II censored sample from $G P(\beta, 1)$. The MLEs of $\alpha$ and $\beta$ are 


$$
\begin{aligned}
\widehat{\alpha} & =\frac{n}{\sum_{i=1}^{n}\left(1+r_{i}\right) \log \left(1+\lambda x_{i}\right)}, \\
\widehat{\beta} & =\frac{m}{\sum_{j=1}^{m}\left(1+r_{j}^{\prime}\right) \log \left(1+\lambda y_{j}\right)} .
\end{aligned}
$$

It is easy to show that $\left(1+r_{i}\right) \log \left(1+\lambda x_{i}\right)$ is an exponential random variable with mean $\frac{1+r_{i}}{\alpha}$. So,

$$
\sum_{i=1}^{n}\left(1+r_{i}\right) \log \left(1+\lambda X_{i}\right) \sim \Gamma\left(n, \frac{\alpha}{1+r_{i}}\right)
$$

and

$$
\sum_{j=1}^{m}\left(1+r^{\prime}{ }_{j}\right) \log \left(1+\lambda Y_{j}\right) \sim \Gamma\left(n, \frac{\beta}{1+r^{\prime}{ }_{j}}\right)
$$

Also we can write

$$
\begin{aligned}
& 2 \alpha \sum_{i=1}^{n}\left(1+r_{i}\right) \log \left(1+\lambda X_{i}\right) \sim \chi^{2}(2 n), \\
& 2 \beta \sum_{j=1}^{m}\left(1+r^{\prime}{ }_{j}\right) \log \left(1+\lambda Y_{j}\right) \sim \chi^{2}(2 m) .
\end{aligned}
$$

So,

$$
\widehat{R}=\frac{1}{\frac{\alpha}{\beta} F+1}
$$

with

$$
\frac{R}{1-R} \times \frac{1-\widehat{R}}{\widehat{R}} \sim F,
$$

an $F$ random variable with $2 m$ and $2 n$ degrees of freedom.

\subsection{UMVUE of $R$}

In Sect. 4.1, we saw that $T^{x}=\log (1+\lambda X)$ is an exponential random variable with mean $\frac{1}{\alpha}$. The joint pdf of $\left(T_{1: N}^{x}, T_{2: N}^{x}, \ldots, T_{n: N}^{x}\right)$ is

$$
f_{T_{1: N}^{x}, \ldots, T_{n: N}^{x}}\left(t_{1: N}^{x}, \ldots, t_{n: N}^{x}\right)=K_{1} \alpha^{n} \exp \left(-\alpha\left(\sum_{i=1}^{n}\left(r_{i}+1\right) t_{i: N}^{x}\right)\right)
$$


for $0<t_{1}^{x}<\cdots<t_{n}^{x}<\infty$, where $K_{1}$ is as defined in (6). Consider the following transformation

$$
\begin{gathered}
Z_{1}^{x}=N T_{1: N}^{x}, \\
Z_{i}^{x}=\left(n-r_{1}-\cdots-r_{n-1}-i+1\right)\left(T_{i: N}^{x}-T_{i-1: N}^{x}\right)
\end{gathered}
$$

for $i=1,2, \ldots, n$. [25] have shown that $Z_{i}$ s are independent exponential random variables with mean $\frac{1}{\alpha}$. Furthermore,

$$
\sum_{i=1}^{n}\left(r_{i}+1\right) T_{i: n}^{x}=\sum_{i=1}^{n} Z_{i}^{x}
$$

and (54) shows that the left hand side is a complete sufficient statistic for $\alpha$.

Theorem 3 The uniformly minimum variance unbiased estimator of $R$ is

$$
\widehat{R}= \begin{cases}1-\int_{0}^{1}(n-1)\left(1-u_{1}\right)^{n-2}\left(1-u_{1} W\right)^{m-1} d u_{1}, & \text { if } W \leq 1 \\ 1-\int_{0}^{1 / W}(n-1)\left(1-u_{1}\right)^{n-2}\left(1-u_{1} W\right)^{m-1} d u_{1}, & \text { if } W>1\end{cases}
$$

Proof An unbiased estimator for $R$ is

$$
\Phi\left(T_{1}, T_{2}\right)= \begin{cases}1, & \text { if } M T_{1: M}^{y}>N T_{2: N}^{x} \\ 0, & \text { if } M T_{1: M}^{y}<N T_{2: N}^{x}\end{cases}
$$

Since $\widehat{R}=E\left(\Phi\left(T_{1}, T_{2}\right) \mid S\right)=P\left(M T_{j ; n}^{y}<N T_{i: n}^{x} \mid S\right)$,

$$
S=\left(S_{1}, S_{2}\right)=\left(\sum_{i=1}^{n}\left(r_{i}+1\right) T_{i: n}^{x}, \sum_{j=1}^{m}\left(r_{j}^{\prime}+1\right) T_{j: n}^{y}\right)
$$

is a jointly completely sufficient statistic. Define

$$
\begin{aligned}
& U_{1}=\frac{Z_{1}^{x}}{\sum_{i=1}^{n} Z_{i}^{x}}, \\
& U_{2}=\frac{Z_{1}^{y}}{\sum_{j=1}^{m} Z_{i}^{y}}, \\
& W=\frac{\sum_{i=1}^{n} Z_{i}^{x}}{\sum_{j=1}^{m} Z_{i}^{y}} .
\end{aligned}
$$


So,

$$
\widehat{R}=P\left(U_{2}<U_{1} W \mid S\right) .
$$

It can be shown that $U_{1}$ and $U_{2}$ are independent beta random variables with parameters $(1, n-1)$ and $(1, m-1)$, respectively, see [5]. Now

$$
f_{U_{1}, U_{2} \mid S}\left(u_{1}, u_{2} \mid S\right)=(n-1)(m-1)\left(1-u_{1}\right)^{n-2}\left(1-u_{2}\right)^{m-2}
$$

for $0<u_{1}, u_{2}<1$. The theorem follows from (64) and (65).

\subsection{Bayes Estimation of $R$}

Here, we assume that $\alpha$ and $\beta$ are random variables. In Bayesian approach, the loss function is important and most studies use the squared error loss (SEL) function, see [7] and [6]. We suppose $\alpha$ and $\beta$ have the gamma priors, i.e., $\pi(\alpha) \sim \Gamma\left(a_{1}, b_{1}\right)$ and $\pi(\beta) \sim \Gamma\left(a_{2}, b_{2}\right)$, where $a_{1}, a_{2}, b_{1}$ and $b_{2}$ are positive constants. Then the posterior pdfs of $\alpha$ and $\beta$ are

$$
\pi(\alpha \mid \text { data }) \sim \Gamma\left(a_{1}+n b_{1}+\sum_{i=1}^{n}\left(1+r_{i}\right) \log \left(1+\lambda x_{i}\right)\right),
$$

and

$$
\pi(\beta \mid \text { data }) \sim \Gamma\left(a_{2}+m b_{2}+\sum_{j=1}^{m}\left(1+r_{j}^{\prime}\right) \log \left(1+\lambda y_{j}\right)\right) .
$$

Using (66) and (67), the posterior pdf of $R$ becomes

$$
f_{R}(r)=k \frac{r^{a_{2}+m-1}(1-r)^{a_{1}+n-1}}{\left\{r\left(b_{2}+S_{2}\right)+(1-r)\left(b_{1}+S_{1}\right)\right\}^{\left(n+m+a_{1}+a_{2}\right)}}
$$

for $0<r<1$, where

$$
k=\frac{\Gamma\left(n+m+a_{1}+a_{2}\right)}{\Gamma\left(n+a_{1}\right) \Gamma\left(m+a_{2}\right)}\left(b_{1}+S_{1}\right)^{a_{1}+n}\left(b_{2}+S_{2}\right)^{a_{2}+m} .
$$

The mean or median of (68) cannot be obtained in closed form. The mode of (68) is the root of

$$
\frac{\partial f_{R}(r)}{\partial r}=\frac{r^{c-1}(1-r)^{c^{\prime}-1}\left\{c^{\prime} d+2 r^{2}\left(d^{\prime}-d\right)+r\left(2 d_{1}-2 d^{\prime}-c^{\prime} d-c d^{\prime}\right)\right\}}{\left\{d(1-r)+d^{\prime} r\right\}^{c+c^{\prime}+3}}=0,
$$


where $c=a_{1}+n-1, c^{\prime}=a_{2}+m-1, d=b_{1}+S_{1}$ and $d^{\prime}=b_{2}+S_{2}$. The mode of the posterior is a Bayes estimator of $R$ with respect to the $0-1$ loss function. Using [14]'s approximation, a Bayes estimator for $R$ under the SEL is

$$
\widehat{R}_{\text {Lindley }}=\widetilde{R}\left\{1+\frac{\widetilde{\alpha} \widetilde{R}^{2}\left[\widetilde{\alpha}\left(n+a_{1}-1\right)-\widetilde{\beta}\left(m+a_{2}-2\right)\right]}{\widetilde{\beta}^{2}\left(n+a_{1}-1\right)\left(m+a_{2}-1\right)}\right\},
$$

where $\widetilde{R}=\frac{\widetilde{\beta}}{\widetilde{\alpha}+\beta}, \widetilde{\alpha}=\frac{n+a_{1}-1}{b_{1}+S_{1}}$, and $\widetilde{\beta}=\frac{m+a_{2}-2}{b_{2}+S_{2}}$.

\subsection{Credible Interval and Confidence Interval of $\boldsymbol{R}$}

Based on asymptotic properties of $R$, we can deduce asymptotic confidence intervals for $R$. Percentile bootstrap and bootstrap- $t$ confidence intervals can also be derived, see Sect. 6.

The credible set for $R$ is given by

$$
P(R \leq L \mid \text { data })=\frac{\gamma}{2} \quad \text { and } \quad P(R \leq U \mid \text { data })=1-\frac{\gamma}{2}
$$

where $L$ and $U$ are the lower and upper bounds. The following algorithm can be used to calculate the credible interval

- Find the mode of the posterior pdf by solving (70), say $r^{m}$.

- generate $k$ random numbers from (68), using the acceptance rejection principle, say $r_{1}, r_{2}, \ldots, r_{k}$, see [8].

- sort $r_{1}, r_{2}, \ldots, r_{k}$ and compute the $\frac{\gamma}{2}$ th and $\left(1-\frac{\gamma}{2}\right)$ th percentiles.

We refer the readers to [23] for other algorithms.

\section{Estimation of $R$ in the General Case}

Let $X \sim G P\left(\alpha, \lambda_{1}\right)$ and $Y \sim G P\left(\beta, \lambda_{2}\right)$ be independent random variables. Then

$$
R=1-\alpha\left(\frac{\lambda_{2}}{\lambda_{1}}\right)^{-\beta} \int_{0}^{\infty}(1+t)^{-(\alpha+1)}\left(\frac{\lambda_{1}}{\lambda_{2}}+t\right)^{-\beta} d t
$$

By using

$$
\begin{aligned}
& \int_{0}^{\infty} x^{\nu-1}(\eta+x)^{-\mu}(x+\gamma)^{-Q} d x \\
& \quad=\eta^{-\mu} \gamma^{\nu-Q} B(\nu, \mu-\nu+Q){ }_{2} F_{1}\left(\mu, \nu ; \mu+Q ; 1-\frac{\gamma}{\eta}\right)
\end{aligned}
$$


for $v>0$ and $\mu>v-Q$, where ${ }_{2} F_{1}$ denotes the Gauss hypergeometric function, we can express (73) as

$$
R=1-\frac{\alpha}{\alpha+\beta} \frac{\lambda_{1}}{\lambda_{2}}{ }_{2} F_{1}\left(\alpha+1,1 ; \alpha+\beta+1 ; 1-\frac{\lambda_{1}}{\lambda_{2}}\right)
$$

\subsection{Maximum Likelihood Estimator of $\boldsymbol{R}$}

Let $X_{1: N}, X_{2: N}, \ldots, X_{n: N}$ and $Y_{1: M}, Y_{2: M}, \ldots, Y_{m: M}$ be progressively type II censored samples from $G P\left(\alpha, \lambda_{1}\right)$ and $G P\left(\beta, \lambda_{2}\right)$, respectively. The likelihood function of the parameters is

$$
\begin{aligned}
& L\left(\alpha, \beta, \lambda_{1}, \lambda_{2}\right) \\
& =K_{1} K_{2} \lambda_{1}^{n} \lambda_{2}^{m} \alpha^{n} \beta^{m} \prod_{i=1}^{n}\left(1+\lambda_{1} x_{i}\right)^{-\left(\alpha\left(1+r_{i}\right)+1\right)} \prod_{j=1}^{m}\left(1+\lambda_{2} y_{j}\right)^{-\left(\beta\left(1+r_{j}^{\prime}\right)+1\right)} .
\end{aligned}
$$

The log likelihood function is

$$
\begin{aligned}
\ell(\alpha, \beta, \lambda)= & \log K_{1}+\log K_{2}+n \log \lambda_{1}+m \log \lambda_{2}+n \log \alpha+m \log \beta \\
& -\sum_{i=1}^{n}\left(\alpha\left(1+r_{i}\right)+1\right) \log \left(1+\lambda_{1} x_{i}\right) \\
& -\sum_{j=1}^{m}\left(\beta\left(1+r_{j}^{\prime}\right)+1\right) \log \left(1+\lambda_{2} y_{j}\right)
\end{aligned}
$$

The MLEs of $\alpha$ and $\beta$ are

$$
\begin{aligned}
\widehat{\alpha} & =\frac{n}{\sum_{i=1}^{n}\left(1+r_{i}\right) \log \left(1+\lambda_{1} x_{i}\right)}, \\
\widehat{\beta} & =\frac{m}{\sum_{j=1}^{m}\left(1+r_{j}^{\prime}\right) \log \left(1+\lambda_{2} y_{j}\right)} .
\end{aligned}
$$

The MLEs of $\lambda_{1}$ and $\lambda_{2}$ are the solutions of

$$
\frac{n}{\lambda_{1}}-\frac{n}{\sum_{i=1}^{n}\left(1+r_{i}\right) \log \left(1+\lambda_{1} x_{i}\right)} \sum_{i=1}^{n} \frac{\left(1+r_{i}\right) x_{i}}{1+\lambda_{1} x_{i}}-\sum_{i=1}^{n} \frac{x_{i}}{1+\lambda_{1} x_{i}}=0
$$




$$
\frac{m}{\lambda_{2}}-\frac{m}{\sum_{i=1}^{n}\left(1+r_{i}^{\prime}\right) \log \left(1+\lambda_{2} y_{i}\right)} \sum_{i=1}^{m} \frac{\left(1+r_{i}^{\prime}\right) y_{i}}{1+\lambda_{2} y_{i}}-\sum_{i=1}^{m} \frac{y_{i}}{1+\lambda_{2} y_{i}}=0 .
$$

By the invariant property, the MLE of $R$ is

$$
\widehat{R}=1-\frac{\widehat{\alpha}}{\widehat{\alpha}+\widehat{\beta}} \widehat{\lambda}_{1}{ }_{\lambda_{2}} F_{1}\left(\widehat{\alpha}+1,1 ; \widehat{\alpha}+\widehat{\beta}+1 ; 1-\frac{\widehat{\lambda}_{1}}{\widehat{\lambda}_{2}}\right) .
$$

\subsection{Asymptotic Distribution}

Here, we compute the asymptotic distribution of $\boldsymbol{\theta}=\left(\widehat{\alpha}, \widehat{\beta}, \widehat{\lambda}_{1}, \widehat{\lambda}_{2}\right)$. This requires the expected Fisher information matrix, i.e.,

$$
\begin{aligned}
I(\theta)=-\left[\begin{array}{llll}
E\left(\frac{\partial^{2} \ell}{\partial \alpha^{2}}\right) & E\left(\frac{\partial^{2} \ell}{\partial \alpha \partial \beta}\right) & E\left(\frac{\partial^{2} \ell}{\partial \alpha \partial \lambda_{1}}\right) & E\left(\frac{\partial^{2} \ell}{\partial \alpha \partial \lambda_{2}}\right) \\
E\left(\frac{\partial^{2} \ell}{\partial \alpha \partial \beta}\right) & E\left(\frac{\partial^{2} \ell}{\partial \beta^{2}}\right) & E\left(\frac{\partial^{2} \ell}{\partial \beta \partial \lambda_{1}}\right) & E\left(\frac{\partial^{2} \ell}{\partial \beta \partial \lambda_{2}}\right) \\
E\left(\frac{\partial^{2} \ell}{\partial \lambda_{1} \partial \alpha}\right) & E\left(\frac{\partial^{2} \ell}{\partial \lambda_{1} \partial \beta}\right) & E\left(\frac{\partial^{2} \ell}{\partial \lambda_{1}^{2}}\right) & E\left(\frac{\partial^{2} \ell}{\partial \lambda_{1} \partial \lambda_{2}}\right) \\
E\left(\frac{\partial^{2} \ell}{\partial \lambda_{2} \partial \alpha}\right) & E\left(\frac{\partial^{2} \ell}{\partial \lambda_{2} \partial \beta}\right) & E\left(\frac{\partial^{2} \ell}{\partial \lambda_{2} \partial \lambda_{1}}\right) & E\left(\frac{\partial^{2} \ell}{\partial \lambda_{2}^{2}}\right)
\end{array}\right] \\
=\left[\begin{array}{llll}
I_{11} & I_{12} & I_{13} & I_{14} \\
I_{21} & I_{22} & I_{23} & I_{24} \\
I_{31} & I_{32} & I_{33} & I_{34} \\
I_{41} & I_{42} & I_{43} & I_{44}
\end{array}\right] .
\end{aligned}
$$

By using

$$
\int_{0}^{\infty} x^{r-1}(1+\gamma x)^{-\delta} d x=\gamma^{-r} B(r, \delta-r)
$$

for $0<r<\delta$, we obtain

$$
\begin{gathered}
I_{11}=\frac{n}{\alpha^{2}}, \\
I_{12}=I_{21}=0, \\
I_{22}=\frac{m}{\beta^{2}}, \\
I_{13}=I_{31}=\frac{\alpha}{\lambda_{1}} \sum_{i=1}^{n}\left(1+r_{i}\right) \sum_{k=1}^{i} a_{k, i} c_{i-1} B\left(2, \alpha \gamma_{k}\right), \\
I_{14}=I_{41}=0, \\
I_{22}=\frac{m}{\beta^{2}},
\end{gathered}
$$




$$
\begin{gathered}
I_{23}=I_{32}=0, \\
I_{24}=I_{42}=\frac{\beta}{\lambda_{2}} \sum_{j=1}^{m}\left(1+r_{j}^{\prime}\right) c_{i-1} \sum_{k=1}^{i} a_{k, i} B\left(2, \beta \gamma_{k}\right), \\
I_{33}=\frac{1}{\lambda_{1}^{2}}\left[n-\alpha \sum_{i=1}^{n}\left(\alpha\left(1+r_{i}\right)+1\right) c_{i-1} \sum_{k=1}^{i} a_{k, i} B\left(3, \alpha \gamma_{k}\right)\right], \\
I_{44}=\frac{1}{\lambda_{2}^{2}}\left[m-\beta \sum_{j=1}^{m}\left(\beta\left(1+r_{j}^{\prime}\right)+1\right) c_{i-1} \sum_{k=1}^{i} a_{k, i} B\left(3, \beta \gamma_{k}\right)\right], \\
I_{34}=I_{43}=0 .
\end{gathered}
$$

Based on the Fisher information matrix, the percentile bootstrap and bootstrap- $t$ confidence intervals can be derived, see Sect. 6 .

\subsection{Bayes Estimation of $\boldsymbol{R}$}

Here, we consider gamma priors for $\alpha, \beta, \lambda_{1}$ and $\lambda_{2}$. We take $\pi(\alpha) \sim \Gamma\left(a_{1}, b_{1}\right)$, $\pi(\beta) \sim \Gamma\left(a_{2}, b_{2}\right), \pi\left(\lambda_{1}\right) \sim \Gamma\left(a_{3}, b_{3}\right)$ and $\pi\left(\lambda_{2}\right) \sim \Gamma\left(a_{4}, b_{4}\right)$, where $a_{1}, a_{2}, a_{3}, a_{4}$ and $b_{1}, b_{2}, b_{3}, b_{4}$ are all positive constants. Some routine algebra shows that the posterior pdfs of $\alpha$ and $\beta$ are

$$
\pi(\alpha \mid \text { data }) \sim \Gamma\left(a_{1}+n, b_{1}+\sum_{i=1}^{n}\left(1+r_{i}\right) \log \left(1+\lambda_{1} x_{i}\right)\right),
$$

and

$$
\pi(\beta \mid \text { data }) \sim \Gamma\left(a_{2}+m b_{2}+\sum_{j=1}^{m}\left(1+r_{j}^{\prime}\right) \log \left(1+\lambda_{2} y_{j}\right)\right)
$$

For $\lambda_{1}$ and $\lambda_{2}$, we see

$$
\pi\left(\lambda_{1} \mid \text { data }\right) \sim \lambda_{1}^{n+a_{3}-1} e^{-\lambda_{1} b_{3}}\left[\prod_{i=1}^{n}\left(1+\lambda_{1} x_{i}\right)^{-(\alpha+1)}\left(1+\lambda_{1} x_{i}\right)^{-\alpha r_{i}}\right]
$$

and 
Table 1 Censoring schemes

\begin{tabular}{lll}
\hline & $(n, N)$ & Censoring scheme \\
\hline$r_{1}$ & $(10,30)$ & $(0,0,0,0,0,0,0,0,0,20)$ \\
$r_{2}$ & $(10,30)$ & $(20,0,0,0,0,0,0,0,0,0)$ \\
$r_{3}$ & $(10,30)$ & $(2,2,2,2,2,2,2,2,2,2)$ \\
\hline
\end{tabular}

Table 2 Information in the case of constant mean

\begin{tabular}{lrrll}
\hline & $a_{i}$ & $b_{i}$ & $\mathrm{E}(\alpha)$ & $\operatorname{Var}(\alpha)$ \\
\hline Least informative & 1 & 2 & 0.5 & 0.25 \\
Informative & 5 & 10 & 0.5 & 0.05 \\
Most informative & 10 & 20 & 0.5 & 0.005 \\
\hline
\end{tabular}

Table 3 Estimation of parameters when $\lambda$ is unknown

\begin{tabular}{|c|c|c|c|c|c|c|c|c|c|}
\hline \multirow[t]{2}{*}{$(\alpha, \beta, \lambda)$} & \multirow[t]{2}{*}{ C.S. } & \multicolumn{2}{|l|}{ P.E. } & \multicolumn{6}{|l|}{ I.E. } \\
\hline & & Bias & MSE & Boot-p & C.P & Boot-t & C.P. & C.I. & C.P. \\
\hline \multirow[t]{6}{*}{$(5,5,1)$} & $\left(r_{1}, r_{1}\right)$ & 0.0250 & 0.0108 & 0.392 & 0.939 & 0.398 & 0.955 & 0.380 & 0.927 \\
\hline & $\left(r_{1}, r_{2}\right)$ & 0.0201 & 0.0060 & 0.390 & 0.946 & 0.395 & 0.950 & 0.375 & 0.925 \\
\hline & $\left(r_{1}, r_{3}\right)$ & 0.0172 & 0.0890 & 0.376 & 0.951 & 0.387 & 0.962 & 0.364 & 0.928 \\
\hline & $\left(r_{2}, r_{2}\right)$ & 0.0071 & 0.0053 & 0.373 & 0.951 & 0.379 & 0.960 & 0.351 & 0.949 \\
\hline & $\left(r_{2}, r_{3}\right)$ & 0.0064 & 0.0061 & 0.364 & 0.941 & 0.369 & 0.955 & 0.351 & 0.936 \\
\hline & $\left(r_{3}, r_{3}\right)$ & 0.0016 & 0.0058 & 0.392 & 0.936 & 0.394 & 0.952 & 0.380 & 0.933 \\
\hline \multirow[t]{6}{*}{$(3,3,2)$} & $\left(r_{1}, r_{1}\right)$ & 0.0261 & 0.0110 & 0.346 & 0.951 & 0.350 & 0.952 & 0.330 & 0.939 \\
\hline & $\left(r_{1}, r_{2}\right)$ & 0.0193 & 0.0091 & 0.330 & 0.945 & 0.340 & 0.953 & 0.321 & 0.949 \\
\hline & $\left(r_{1}, r_{3}\right)$ & 0.0163 & 0.0070 & 0.324 & 0.949 & 0.340 & 0.956 & 0.310 & 0.945 \\
\hline & $\left(r_{2}, r_{2}\right)$ & 0.0088 & 0.0058 & 0.318 & 0.941 & 0.327 & 0.953 & 0.302 & 0.940 \\
\hline & $\left(r_{2}, r_{3}\right)$ & 0.0070 & 0.0050 & 0.311 & 0.947 & 0.322 & 0.950 & 0.294 & 0.940 \\
\hline & $\left(r_{3}, r_{3}\right)$ & 0.0183 & 0.0103 & 0340 & 0.951 & 0.348 & 0.955 & 0.326 & 0.942 \\
\hline \multirow[t]{6}{*}{$(7,7,2 / 3)$} & $\left(r_{1}, r_{1}\right)$ & 0.0151 & 0.0106 & 0.276 & 0.952 & 0.288 & 0.951 & 0.261 & 0.940 \\
\hline & $\left(r_{1}, r_{2}\right)$ & 0.0117 & 0.0066 & 0.256 & 0.945 & 0.263 & 0.956 & 0.247 & 0.953 \\
\hline & $\left(r_{1}, r_{3}\right)$ & 0.0092 & 0.0041 & 0.240 & 0.948 & 0.250 & 0.952 & 0.229 & 0.950 \\
\hline & $\left(r_{2}, r_{2}\right)$ & 0.0089 & 0.0046 & 0.221 & 0.950 & 0.233 & 0.960 & 0.204 & 0.943 \\
\hline & $\left(r_{2}, r_{3}\right)$ & 0.0070 & 0.0063 & 0.207 & 0.947 & 0.215 & 0.960 & 0.202 & 0.938 \\
\hline & $\left(r_{3}, r_{3}\right)$ & 0.0039 & 0.0090 & 0.260 & 0.944 & 0.271 & 0.949 & 0.239 & 0.938 \\
\hline
\end{tabular}

$$
\pi\left(\lambda_{2} \mid \text { data }\right) \sim \lambda_{2}^{m+a_{4}-1} e^{-\lambda_{2} b_{4}}\left[\prod_{j=1}^{m}\left(1+\lambda_{2} y_{j}\right)^{-(\beta+1)}\left(1+\lambda_{2} y_{j}\right)^{-\beta r_{j}^{\prime}}\right]
$$

The Metropolis-Hastings simulation method can be used to obtain the posterior pdfs of $\lambda_{1}$ and $\lambda_{2}$. We refer the readers to [23] for other algorithms. 
Table 4 Bayes estimation of parameters when $(\alpha=3, \beta=3, \lambda=1)$

Table 5 Bayes estimation of parameters when $(\alpha=5, \beta=5, \lambda=1)$

\begin{tabular}{lllll}
\hline Information & C.S. & E.R. & H.P.D. & C.P. \\
\hline Least informative & $\left(r_{1}, r_{1}\right)$ & 0.0119 & 0.355 & 0.948 \\
& $\left(r_{1}, r_{2}\right)$ & 0.0091 & 0.353 & 0.941 \\
& $\left(r_{1}, r_{3}\right)$ & 0.0129 & 0.348 & 0.940 \\
& $\left(r_{2}, r_{2}\right)$ & 0.0083 & 0.340 & 0.951 \\
& $\left(r_{2}, r_{3}\right)$ & 0.0085 & 0.335 & 0.954 \\
Informative & $\left(r_{3}, r_{3}\right)$ & 0.0090 & 0.352 & 0.933 \\
& $\left(r_{1}, r_{1}\right)$ & 0.0102 & 0.351 & 0.947 \\
& $\left(r_{1}, r_{2}\right)$ & 0.0086 & 0.346 & 0.940 \\
& $\left(r_{1}, r_{3}\right)$ & 0.0120 & 0.343 & 0.939 \\
& $\left(r_{2}, r_{2}\right)$ & 0.0081 & 0.334 & 0954 \\
& $\left(r_{2}, r_{3}\right)$ & 0.0081 & 0.331 & 0.942 \\
& $\left(r_{3}, r_{3}\right)$ & 0.0088 & 0.344 & 0.930 \\
& $\left(r_{1}, r_{1}\right)$ & 0.0093 & 0.334 & 0.951 \\
& $\left(r_{1}, r_{2}\right)$ & 0.0076 & 0.332 & 0.948 \\
& $\left(r_{1}, r_{3}\right)$ & 0.0114 & 0.338 & 0.939 \\
& $\left(r_{2}, r_{2}\right)$ & 0.0075 & 0.330 & 0.950 \\
& $\left(r_{2}, r_{3}\right)$ & 0.0080 & 0.319 & 0.940 \\
$\left(r_{3}, r_{3}\right)$ & 0.0077 & 0.334 & 0.930 \\
& & & &
\end{tabular}

\begin{tabular}{|c|c|c|c|c|}
\hline Information & C.S. & E.R. & H.P.D. & C.P. \\
\hline \multirow[t]{6}{*}{ Least informative } & $\left(r_{1}, r_{1}\right)$ & 0.0132 & 0.319 & 0.946 \\
\hline & $\left(r_{1}, r_{2}\right)$ & 0.0115 & 0.316 & 0.949 \\
\hline & $\left(r_{1}, r_{3}\right)$ & 0.0093 & 0.304 & 0.958 \\
\hline & $\left(r_{2}, r_{2}\right)$ & 0.0088 & 0.290 & 0.952 \\
\hline & $\left(r_{2}, r_{3}\right)$ & 0.0077 & 0.279 & .0955 \\
\hline & $\left(r_{3}, r_{3}\right)$ & 0.0126 & 0.320 & 0.948 \\
\hline \multirow[t]{6}{*}{ Informative } & $\left(r_{1}, r_{1}\right)$ & 0.0120 & 0.317 & 0.945 \\
\hline & $\left(r_{1}, r_{2}\right)$ & 0.0110 & 0.310 & 0.949 \\
\hline & $\left(r_{1}, r_{3}\right)$ & 0.0080 & 0.301 & 0.954 \\
\hline & $\left(r_{2}, r_{2}\right)$ & 0.0081 & 0.279 & 0.948 \\
\hline & $\left(r_{2}, r_{3}\right)$ & 0.0068 & 0.280 & 0.951 \\
\hline & $\left(r_{3}, r_{3}\right)$ & 0.124 & 0.310 & 0.952 \\
\hline \multirow[t]{6}{*}{ Most informative } & $\left(r_{1}, r_{1}\right)$ & 0.0116 & 0.302 & 0.944 \\
\hline & $\left(r_{1}, r_{2}\right)$ & 0.0103 & 0.301 & 0.944 \\
\hline & $\left(r_{1}, r_{3}\right)$ & 0.0076 & 0.292 & 0.951 \\
\hline & $\left(r_{2}, r_{2}\right)$ & 0.0072 & 0.280 & 0.949 \\
\hline & $\left(r_{2}, r_{3}\right)$ & .0060 & 0.277 & 0.946 \\
\hline & $\left(r_{3}, r_{3}\right)$ & 0.0110 & 0.309 & 0.940 \\
\hline
\end{tabular}


Table 6 Bayes estimation of parameters when $(\alpha=7, \beta=7, \lambda=1)$

\begin{tabular}{|c|c|c|c|c|}
\hline Information & C.S. & E.R. & H.P.D. & C.P. \\
\hline \multirow[t]{6}{*}{ Least informative } & $\left(r_{1}, r_{1}\right)$ & 0.0133 & 0.254 & 0.952 \\
\hline & $\left(r_{1}, r_{2}\right)$ & 0.109 & 0.240 & 0.954 \\
\hline & $\left(r_{1}, r_{3}\right)$ & 0.0061 & 0.220 & 0.950 \\
\hline & $\left(r_{2}, r_{2}\right)$ & 0.0071 & 0.211 & 0.948 \\
\hline & $\left(r_{2}, r_{3}\right)$ & 0.0110 & 0.0203 & 0.942 \\
\hline & $\left(r_{3}, r_{3}\right)$ & 0.0130 & 0.239 & 0.951 \\
\hline \multirow[t]{6}{*}{ Informative } & $\left(r_{1}, r_{1}\right)$ & 0.0129 & 0.250 & 0.939 \\
\hline & $\left(r_{1}, r_{2}\right)$ & 0.0100 & 0.240 & 0.951 \\
\hline & $\left(r_{1}, r_{3}\right)$ & 0.0054 & 0.209 & 0.954 \\
\hline & $\left(r_{2}, r_{2}\right)$ & 0.0060 & 0.202 & 0.959 \\
\hline & $\left(r_{2}, r_{3}\right)$ & 0.0099 & 0.177 & 0.943 \\
\hline & $\left(r_{3}, r_{3}\right)$ & 0.0127 & 0.244 & 0.950 \\
\hline \multirow[t]{6}{*}{ Most informative } & $\left(r_{1}, r_{1}\right)$ & 0.0118 & 0.233 & 0.941 \\
\hline & $\left(r_{1}, r_{2}\right)$ & 0.0804 & 0.230 & 0.942 \\
\hline & $\left(r_{1}, r_{3}\right)$ & 0.0053 & 0.213 & 0.946 \\
\hline & $\left(r_{2}, r_{2}\right)$ & 0.0055 & 0.197 & 0.949 \\
\hline & $\left(r_{2}, r_{3}\right)$ & 0.0084 & 0.184 & 0.949 \\
\hline & $\left(r_{3}, r_{3}\right)$ & 0.0116 & 0.240 & 0.945 \\
\hline
\end{tabular}

Table 7 Estimation of parameters in the exponential $(\mu=0, \xi=0, \sigma=1)$ and Pareto $\left(\frac{1}{\xi}=2, \frac{\sigma}{\xi}=4\right)$ distributions

\begin{tabular}{|c|c|c|c|c|c|c|}
\hline$(\mu, \xi, \sigma)$ & C.S. & Bias & MSE & ER & E.C.I & C.P. \\
\hline \multirow[t]{6}{*}{ Exponential } & $\left(r_{1}, r_{1}\right)$ & 0.0018 & 0.0123 & 0.0109 & 0.399 & 0.948 \\
\hline & $\left(r_{1}, r_{2}\right)$ & 0.0015 & 0.0118 & 0.0110 & 0.399 & 0.949 \\
\hline & $\left(r_{1}, r_{3}\right)$ & 0.0010 & 0.0117 & 0.0098 & 0.400 & 0.955 \\
\hline & $\left(r_{2}, r_{2}\right)$ & 0.0021 & 0.0121 & 0.0108 & 0.399 & 0.943 \\
\hline & $\left(r_{2}, r_{3}\right)$ & 0.0029 & 0.0112 & 0.0100 & 0.403 & 0.957 \\
\hline & $\left(r_{3}, r_{3}\right)$ & 0.0037 & 0.0122 & 0.0109 & 0.401 & 0.951 \\
\hline \multirow[t]{6}{*}{ Pareto } & $\left(r_{1}, r_{1}\right)$ & 0.0030 & 0.0121 & 0.0112 & 0.401 & 0.947 \\
\hline & $\left(r_{1}, r_{2}\right)$ & 0.0024 & 0.0117 & 0.0113 & 0.402 & 0.947 \\
\hline & $\left(r_{1}, r_{3}\right)$ & 0.0011 & 0.0114 & 0.0094 & 0.402 & 0.947 \\
\hline & $\left(r_{2}, r_{2}\right)$ & 0.0071 & 0.0119 & 0.0103 & 0.404 & 0.953 \\
\hline & $\left(r_{2}, r_{3}\right)$ & 0.0025 & 0.0120 & 0.0101 & 0.401 & 0.945 \\
\hline & $\left(r_{3}, r_{3}\right)$ & 0.0027 & 0.0120 & 0.0107 & 0.401 & 0.943 \\
\hline
\end{tabular}

\section{Simulation Study}

Here, a Monte Carlo simulation study comparing the performances of all the estimators in the preceding sections is presented. We use the three censoring schemes in Table 1, those used in [26]. 
The estimators are compared in terms of bias, MSE, coverage probability, coverage length and estimated risk. The priors for $\left(\alpha, \beta, \lambda_{1}, \lambda_{2}\right)$ were taken to be one of least informative, informative or most informative, see Table 2 (Tables 3, 4, 5, 6, 7).

\section{Conclusions}

In this paper, we have addressed the problem of estimating $R=P(Y<X)$ for the generalized Pareto distribution based on progressively censored samples. We have considered the cases when the common scale parameter is known or unknown and when the scale parameters are different. When the common scale parameter is unknown, the maximum likelihood estimator works quite well. We have used the asymptotic distribution of the maximum likelihood estimator to construct confidence intervals. These intervals work well even for small sample sizes. Based on simulation results, we recommend to use the parametric bootstrap percentile method when the sample size is very small.

We have observed that the Bayes estimator has the smallest estimated risk. The scheme $\left(r_{1}, r_{3}\right)$ has smaller MSE compared to the other schemes. Also in most cases the confidence interval for the $\left(r_{1}, r_{3}\right)$ scheme has the shortest length. The estimated risk decreases as the priors become more "informative".

Acknowledgments The authors would like to thank the Editor and the referee for careful reading and comments which greatly improved the paper.

\section{References}

1. Babayi S, Khorrama E, Tondro F (2014) Inference of $R=P[X<Y]$ for generalized logistic distribution. Statistics 48:862-871

2. Balakrishnan N (2007) Progressive censoring methodology: an appraisal (with discussions). TEST 16:211-296

3. Balakrishnan N, Aggarwala R (2000) Progressive censoring: theory, methods, and applications. Springer, New York

4. Baklizi A, El-Masri AQ (2004) Shrinkage estimation of $P(X<Y)$ in the exponential case with common location parameter. Metrika 59:163-171

5. Basu D (1955) On statistics independent of a complete sufficient statistic. Sankhyā 15:377-380

6. Berger JO (1985) Statistical decision theory and bayesian analysis. Springer, New York

7. Box GE, Tiao GC (2011) Bayesian inference in statistical analysis. Wiley, New York

8. Devroye L (1984) A simple algorithm for generating random variates with a logconcave density function. Computing 33:247-257

9. Kamps U, Cramer E (2001) On distributions of generalized order statistics. Statistics 35:269-280

10. Kotz S, Lumelskii Y, Pensky M (2003) The stress-strength model and its generalizations: theory and applications. World Scientific Publishing, Singapore

11. Krishnamoorthy K, Mukherjee S, Guo H (2007) Inference on reliability in two-parameter exponential stressstrength model. Metrika 65:261-273

12. Kundu D, Gupta RD (2005) Estimation of $\mathrm{P}[\mathrm{Y}<\mathrm{X}]$ for generalized exponential distribution. Metrika 61:291-308

13. Kundu D, Gupta D (2006) Estimation of $\mathrm{P}(\mathrm{Y}<\mathrm{X})$ for Weibull distribution. IEEE Trans Reliab 55:270280

14. Lindley DV (1980) Approximation bayesian methods. Trabajos de Estadistica 21:223-237

15. Mokhlis NA (2005) Reliability of a stressstrength model with Burr type III distributions. Commun Stat Theo Methods 34:1643-1657 
16. Nadarajah S (2003) Reliability for life time distributions. Math Comput Model 37:683-688

17. Nadarajah S (2004a) Reliability for logistic distributions. Elektronnoe Modelirovanie 26:65-82

18. Nadarajah S (2004b) Reliability for Laplace distributions. Math Prob Eng 2004(2):169-183

19. Nadarajah S (2005a) Reliability for some bivariate beta distributions. Math Prob Eng 2005(1):101-111

20. Nadarajah S (2005) Reliability for some bivariate gamma distributions. Math Prob Eng 2005(2):151163

21. Nadarajah S, Kotz S (2006) Reliability for some bivariate exponential distributions. Math Prob Eng 2006:1-14

22. Raqab MZ, Madi MT, Kundu D (2008) Estimation of $\mathrm{P}(\mathrm{Y}<\mathrm{X})$ for a three-parameter generalized exponential distribution. Commun Stat Theo Methods 37:2854-2864

23. Rezaei S, Tahmasbi R, Mahmoodi M (2010) Estimation of $\mathrm{P}(\mathrm{Y}<\mathrm{X})$ for generalized Pareto distribution. J Stat Plan Inference 140:480-494

24. Saraoglu B, Kaya MF (2007) Maximum likelihood estimation and confidence intervals of system reliability for Gompertz distributions stressstrength models. Seluk J Appl Math 8:25-36

25. Thomas DR, Wilson WM (1972) Linear order statistic estimation for the two-parameter Weibull and extreme value distribution from type II progressively censored samples. Technometrics 14:679-691

26. Valiollahi R, Asgharzadeh A, Raqab MZ (2013) Estimation of $\mathrm{P}(\mathrm{Y}<\mathrm{X})$ for Weibull distribution under progressive type-II censoring. Commun Stat Theo Methods 42:4476-4498 